\title{
A NEW SPATIAL AND TEMPORAL FUSION MODEL
}

\author{
Jing Wang ${ }^{\mathrm{a}}$, Bo Huang ${ }^{\mathrm{a}, *}$ \\ ${ }^{a}$ Institute of Space and Earth Information Science, The Chinese University of Hong Kong, Shatin, Hong Kong - \\ (wangjing1990@link.cuhk.edu.hk, bohuang@cuhk.edu.hk)
}

\author{
Commission VII, WG VII/6
}

KEY WORDS: Auto-regression Error, Image Fusion, Landsat, MODIS, Spatial and Temporal Model

\begin{abstract}
:
As Moderate Resolution Imaging Spectroradiometer (MODIS) and Enhanced Thematic Mapper Plus (ETM+) has a tradeoff between the high temporal resolution and high spatial resolution, this paper proposed a spatial and temporal model with auto-regression error correction (AREC) method to blend the two types of images in order to obtain the composed image with both high spatial and temporal resolution. Experiments and validation were conducted on a data set located in Shenzhen, China and compared with Spatial and Temporal Adaptive Reflectance Fusion Model (STARFM) in several objective indexes and visual analysis. It was found that AREC could effectively predict the land cover changes and the fusion results had better performances versus the ones of STARFM.
\end{abstract}

\section{INTRODUCTION}

Due to sensor technological limitations, the Enhanced Thematic Mapper Plus (ETM+) has low temporal resolution of 16-day revisit cycle but fine spatial resolution of $30 \mathrm{~m}$ while the Moderate Resolution Imaging Spectroradiometer (MODIS), visits the globe once or twice per day with coarse resolution about 250-1000 m. Besides the difference in spatial and temporal resolution, MODIS has corresponding bands to the ETM+ but different bandwidths (Table1). Thus the combination of ETM+ and MODIS data is a necessary and feasible solution to the trade-off between spatial and temporal resolution due to current satellite sensors limits. This paper (Gao et al., 2006) proposed a Spatial and Temporal Adaptive Reflectance Fusion
Model (STARFM) in 2006. The spatial temporal autoregression (STAR) model (Cliff and Ord, 1975; Szummer and Picard, 1996) was a linear model, which also could be introduced to spatial and temporal fusion.

This paper proposes a spatial and temporal model with autoregression error correction (AREC) to predict daily surface reflectance at Landsat spatial resolution. The Shenzhen data set included two pairs of registered Landsat-MODIS images acquired on the same dates in the same region. The results of visual analysis and quantitative evaluation between the AREC and STARFM turn out that the AREC could capture the change information and improve the accuracy of surface reflectance prediction.

\begin{tabular}{|c|c|c|c|c|c|}
\hline \multicolumn{3}{|c|}{ ETM+ } & \multicolumn{3}{|c|}{ MODIS } \\
\hline Band No. & Bandwidth (nm) & Spatial Resolution (m) & Band No. & Bandwidth (nm) & Spatial Resolution (m) \\
\hline 1 & $450-520$ & 30 & 3 & $459-479$ & 500 \\
\hline 2 & $530-610$ & 30 & 4 & $545-565$ & 500 \\
\hline 3 & $630-690$ & 30 & 1 & $620-670$ & 250 \\
\hline 4 & $780-900$ & 30 & 2 & $841-876$ & 250 \\
\hline 5 & $1550-1750$ & 30 & 6 & $1628-1652$ & 500 \\
\hline 7 & $2090-2350$ & 30 & 7 & $2105-2155$ & 500 \\
\hline
\end{tabular}

Table 1. The corresponding bandwidths and spatial resolution in ETM+ and MODIS

\section{METHOD}

This method is based on the spatial temporal auto-regression model. The processing scheme can be summarized as follows. First, we apply an unsupervised classification method to classify the input MODIS images into corresponding clumps. Then we detect the changed area by a threshold method. If an area did not change, we apply the regression model on spatial corresponding pixels. Otherwise, we search the classification result and find the spectrally similar pixels. Following this, we apply the regression model on spectrally similar pixels. Next, in order to improve the detail information and visualization, we perform post-processing such as back projection. Finally, we can generate the fusion result with Landsat resolution and MODIS change information.

\subsection{First step}

Firstly, an unsupervised classification is performed on a pair of MODIS images. This method employs the unsupervised classification (K-Means or IsoData) method to classify the MODIS images into 8-10 categories (according to the specific situation).

\subsection{Second step}

Next land cover change detection is applied and the AREC model is implemented. We adopt thresholds of surface reflectance difference to detect the changes in the images on two different dates. 


$$
\operatorname{dif}(x, y)=\left|M_{2}(x, y)-M_{1}(x, y)\right|
$$

where $\operatorname{dif}(x, y)$ is the absolute value of the surface reflectance difference between MODIS $t_{1}\left(M_{1}\right)$ and MODIS $t_{2}\left(M_{2}\right)$ in a given pixel $(x, y)$. The threshold is set as $\overline{\mathrm{dif}}$, which means the average difference between MODIS images. If the absolute value lies beyond the threshold, the surface is marked as changed area. We search neighbor of the pixel in MODIS $t_{1}$ which belong to the same category of MODIS $t_{2}$. Then we compare the neighbor of every candidate pixel with the neighbor of the pixel in MODIS $t_{2}$ and figure out the most similar one according to the Euclidean distance. Then the autoregression error model (2)(3) is applied to the new spectrally corresponding pixels. In all other cases it means that the surface reflectance is nearly the same with the previous date, and we apply the spatial corresponding pixels by auto-regression error model (2)(3) (Wang et al., 2012).

$$
\begin{aligned}
T_{1, i}(x, y)_{N * N} & =M_{1, i}(x, y)_{N * N} \beta+\mu, \\
\mu & =\rho W \mu+\varepsilon
\end{aligned}
$$

where $T_{1, i}(x, y)_{N * N}$ is the ETM+ image vector of the band $i$ in the local moving window at the reference date $t_{1}, M_{1, i}(x, y)_{N * N}$ is the MODIS image vector of the band $i$ in the corresponding location at the same time $t_{1}$, similarly $M_{2, i}(x, y)_{N * N}$ is the MODIS image vector of the band $i$ in the corresponding location at the different time $t_{2},(x, y)_{N * N}$ is the local area $(N * N)$ in the center of a given pixel $(x, y)$ (omitted in the follow derivation), $\beta$ is regression parameter, $\mu$ is a zero mean auto-regression random error vector, $W$ is the spatial weight matrix, $\varepsilon$ is a vector of independent and identically distributed (IID) random error, $\rho$ is a positively parameter as $\mu$ and $W \mu$ are positively correlated. When the surface reflectance has changed, we suppose that the relationship between MODIS and Landsat of different time are the same in the term of imagery mechanism and system errors. Then, we can get $\varepsilon_{t 1}=\varepsilon_{t 2}$. We can use the relationship between MODIS and Landsat in the time $t_{1}$ and get change information from MODIS time $t_{2}$ to obtain the predict Landsat image namely $T_{2, i}(x, y)_{N * N}=M_{2, i}(x, y)_{N * N} \beta+\mu$.

\subsection{Third step}

The final step uses a back projection (Irani and Peleg, 1991) to MODIS $t_{2}$. We apply the local back prediction method to modify the results. We resample the result to the MODIS resolution and compare the neighbor pixels with the actual MODIS $t_{2}$. Then the difference to the neighbor pixels is reassigned.

\section{EXPERIMENTS}

This paper utilizes a data set to implement the AREC method and compares the result with the STARFM by visualization analysis and selected objective quality indexes.

The data set covers an area in Shenzhen, China, and was acquired on 1 Nov 2000 and 7 Nov 2002 respectively. The image size is $480 * 480 * 3$. We used false color of band 2(Near Infrared), band 1(Red), and band 4 (Green) of MODIS image and band 4 (Near Infrared), band 3(Red) and band 2 (Green) of Landsat image for displaying in Figure 1.

Then we applied the AREC and STARFM method to generate the predicted ETM+ images independently. The visualization of the result is shown below (figure 2).

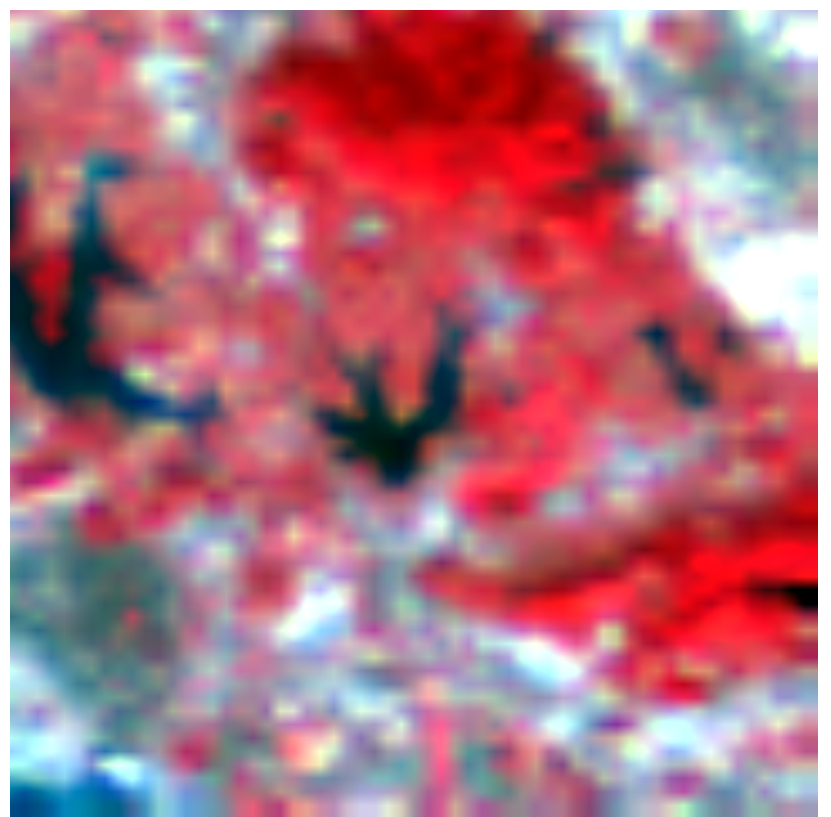

(a)

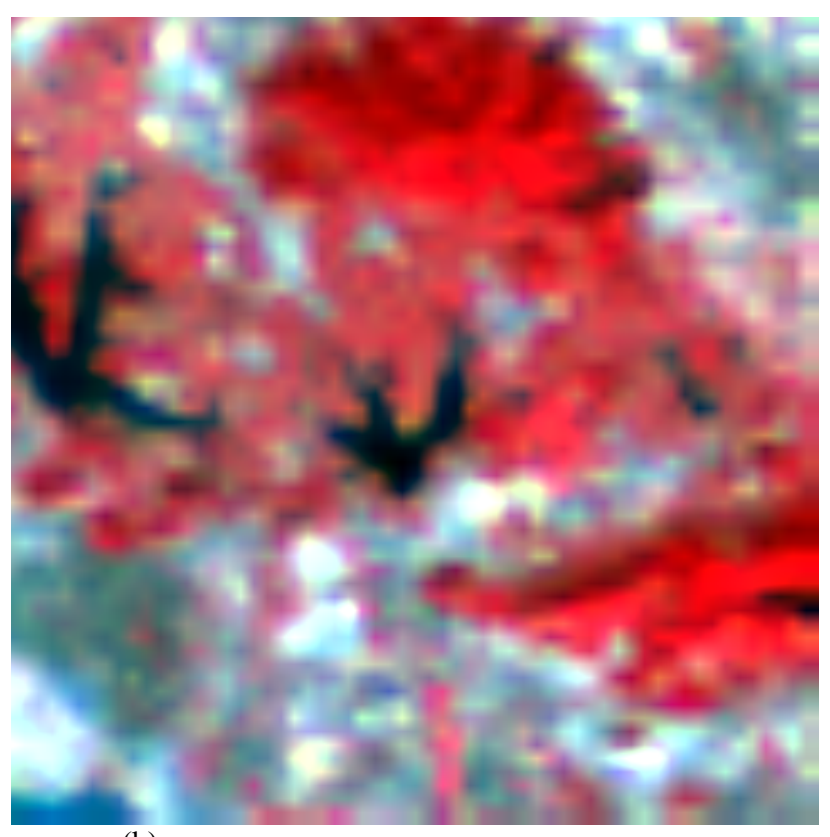

(b) 


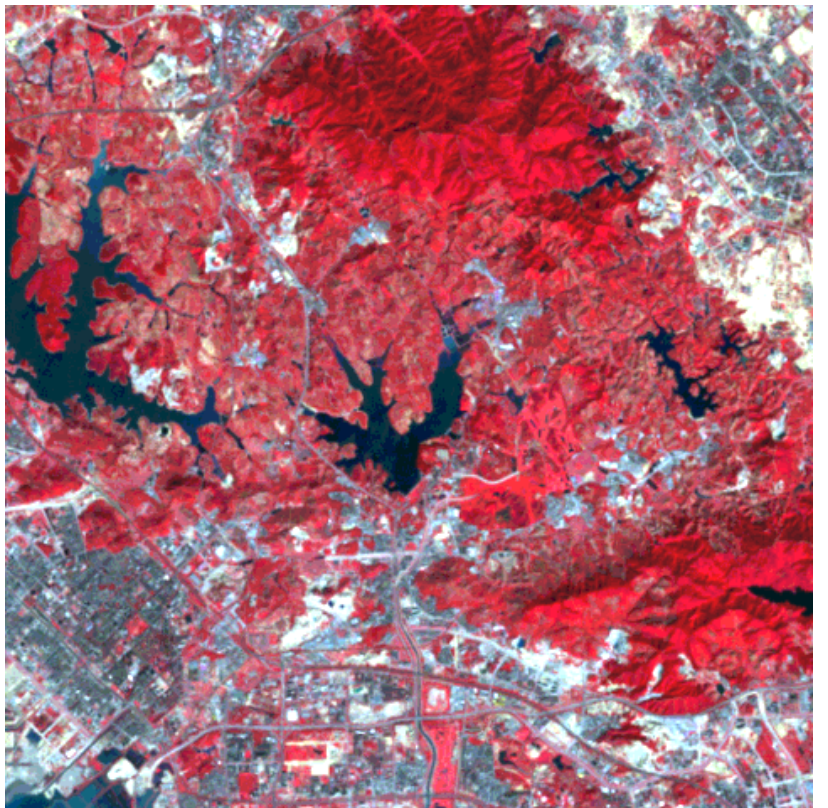

(c)

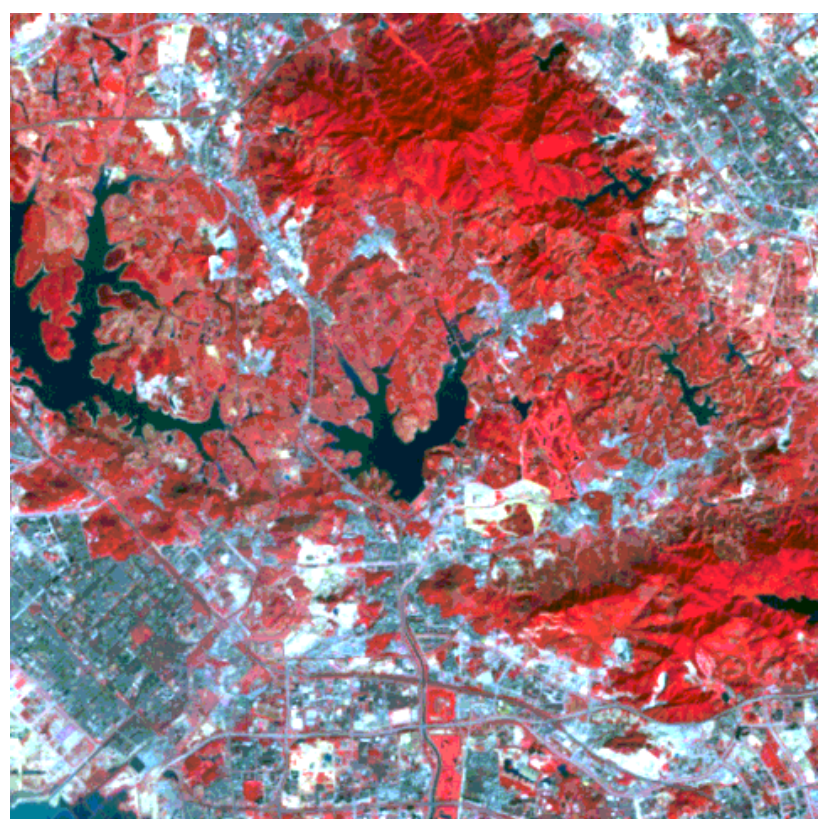

(d)

Figure 1. Shenzhen, China Data Set. The upper two are NIR-red-green composites of MODIS surface reflectance at $250 \mathrm{~m}$ spatial resolution (red band has $500 \mathrm{~m}$ spatial resolution which has been interpolated to $250 \mathrm{~m}$ ), the lower two show corresponding Landsat surface reflectance at $30 \mathrm{~m}$ spatial resolution. (a) MODIS 2000, (b) MODIS 2002, (c) Landsat 2000, (d) Landsat 2002.

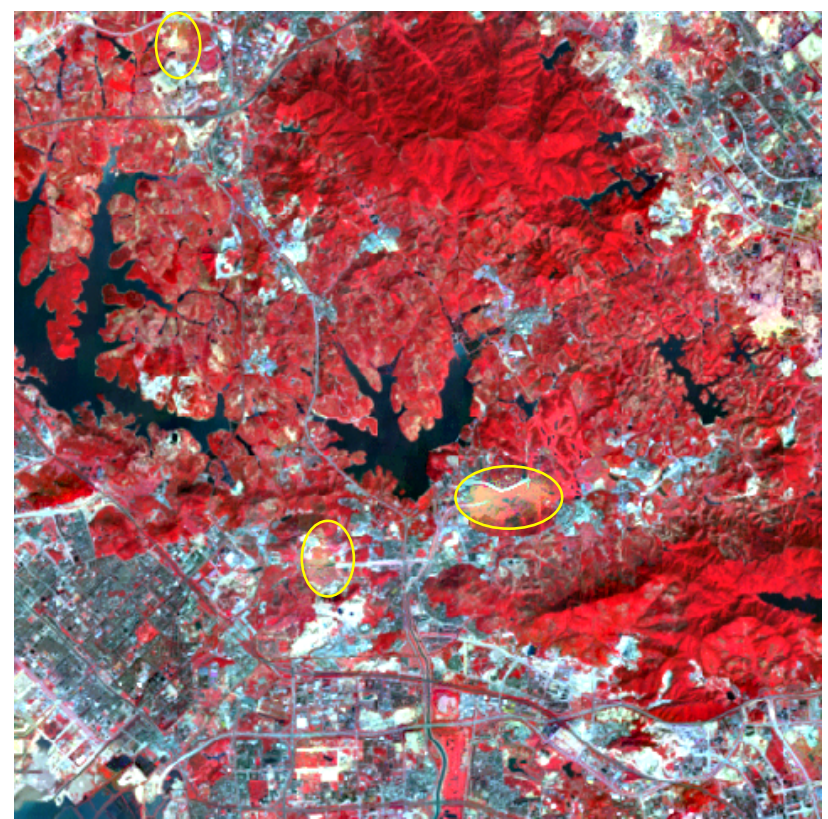

(a)

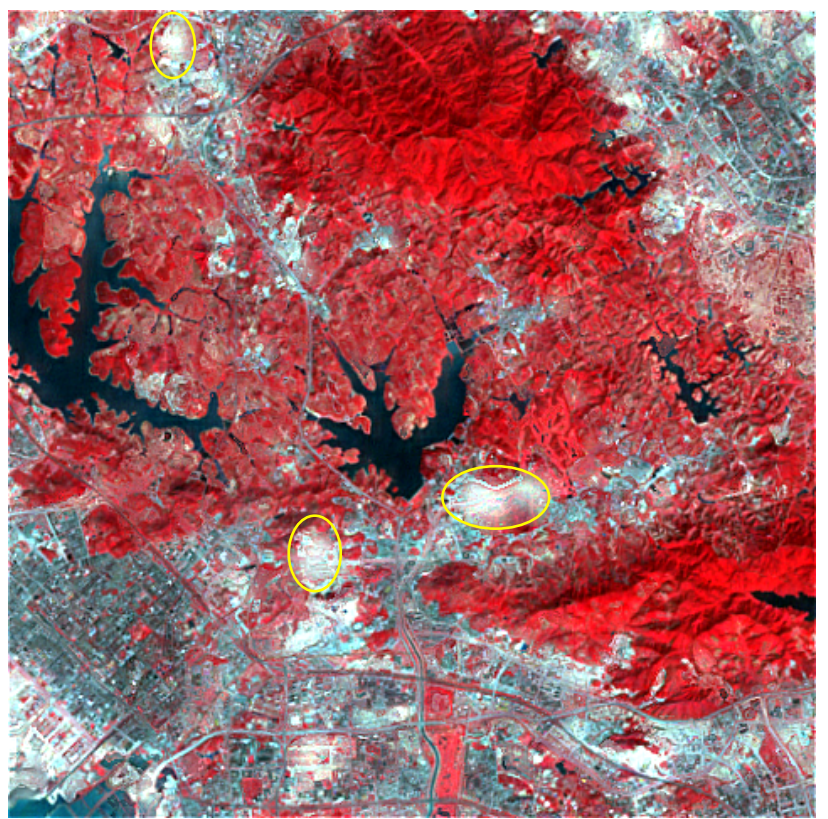

(b)

Figure 2. Comparison of the results by STARFM and AREC methods. (a) STARFM (b) AREC

We can see from Figure 2(a) that the STARFM method could detect part of the land cover changes and preserve the high frequency information. Figure 2(b) shows that the main changes have been detected and the edges and borders are articulated. Scatter plots in Figure 3 show the relationship between the predicted and actual reflectance on 7, Nov 2002 for the three bands respectively. All the scatter points fall close to the diagonal line (1:1). Table 2 evaluates the two methods using quality indexes, namely average absolute differences (AAD), average differences (AD), Root mean square error (RMSE), erreur relative globale adimensionnelle de synthese (ERGAS) (Wald, 2002) and correlation coefficient (CC) in three bands respectively. According to the above indexes, both algorithms have small difference compared with reference one, while the AREC method has a better performance and captures more type changes than STARFM (areas in yellow circles of Figure 2). 

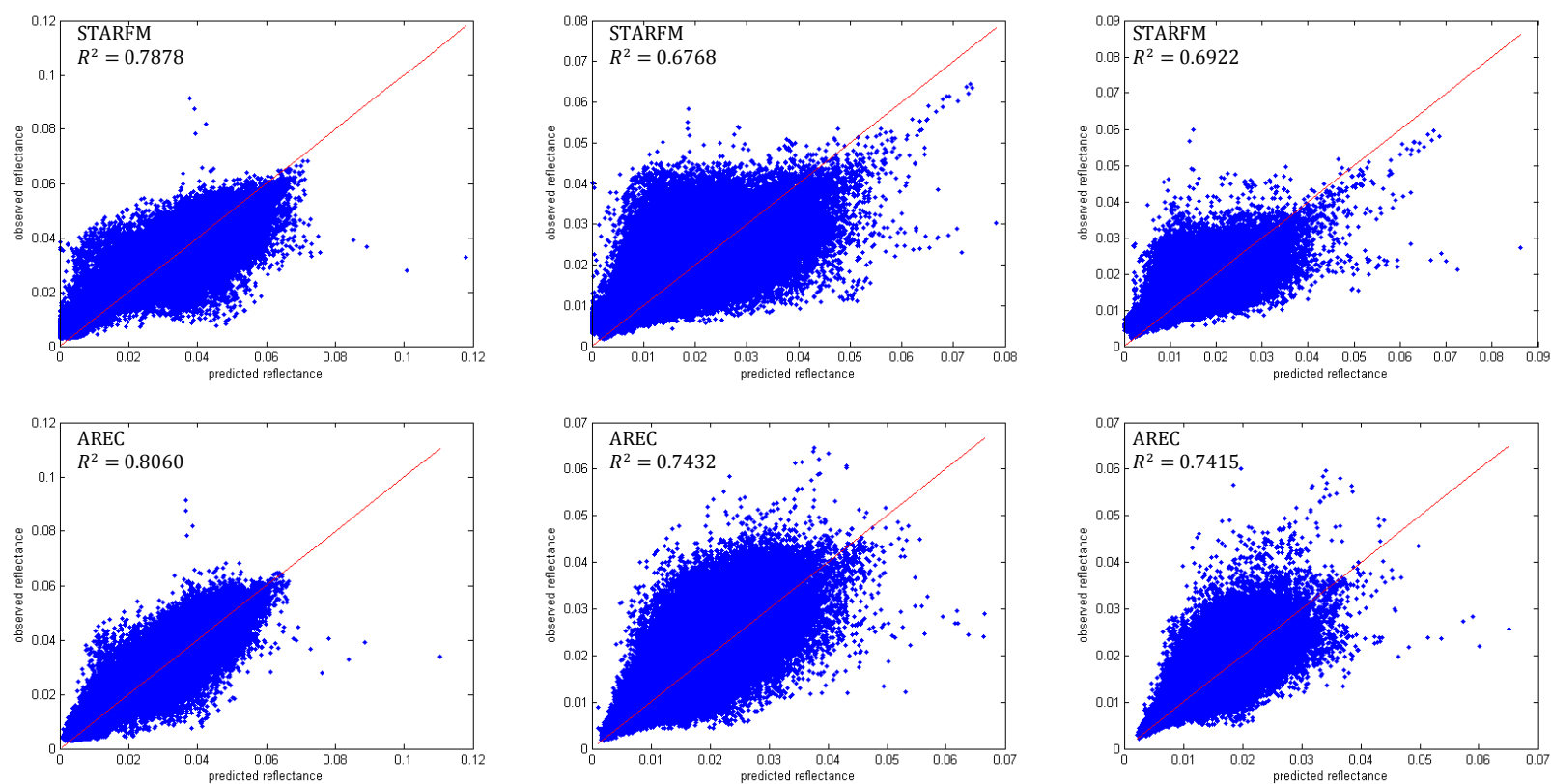

Figure 3. Scatter plots of the predicted reflectance and the real one product by STARFM and AREC for NIR, Red and Green band in Shenzhen data set

\begin{tabular}{|c|c|c|c|c|c|c|}
\hline Method & \multicolumn{3}{|c|}{ AAD $\left(10^{-2}\right)$} & \multicolumn{3}{c|}{ AD $\left(10^{-2}\right)$} \\
\hline & NIR & Red & Green & NIR & Red & Green \\
\hline STARFM & 0.37 & 0.32 & 0.25 & 0.14 & $\mathbf{0 . 0 2}$ & -0.04 \\
\hline AREC & $\mathbf{0 . 3 0}$ & $\mathbf{0 . 2 5}$ & $\mathbf{0 . 1 9}$ & $\mathbf{0 . 0 5}$ & $\mathbf{- 0 . 0 2}$ & $\mathbf{- 0 . 0 3}$ \\
\hline & 0.52 & 0.50 & 0.37 & 0.8876 & 0.8227 & 0.8320 \\
\hline STARFM & $\mathbf{0 . 4 2}$ & $\mathbf{0 . 3 9}$ & $\mathbf{0 . 2 9}$ & $\mathbf{0 . 8 9 7 8}$ & $\mathbf{0 . 8 6 2 1}$ & $\mathbf{0 . 8 6 1 1}$ \\
\hline AREC & \multicolumn{7}{|c|}{ ERGAS } \\
\hline & \multicolumn{7}{|c|}{1.7750} \\
\hline STARFM & \multicolumn{7}{|c|}{$\mathbf{1 . 3 8 5 1}$} \\
\hline AREC & \multicolumn{7}{|c|}{} \\
\hline
\end{tabular}

Table 2. The Shenzhen data set fusion results evaluated by objective indexes for STARFM and AREC methods

\section{CONCLUSIONS}

The experiment results illustrated that this method can obtain a fusion image with the high spatial resolution of ETM+ and high temporal resolution of MODIS image. While this method still have some shortcomings. The details of changed area can not be preserved as well as before. Also this method takes more time than STARFM. This problem occurs when applied into big data sets.

\section{REFERENCES}

Cliff, A.D., Ord, J.K., 1975. Space-Time Modelling with an Application to Regional Forecasting. T I Brit Geogr, pp. 119128.

Gao, F., Masek, J., Schwaller, M., Hall, F., 2006. On the blending of the Landsat and MODIS surface reflectance:
Predicting daily Landsat surface reflectance. Ieee T Geosci Remote 44, pp. 2207-2218.

Irani, M., Peleg, S., 1991. Improving Resolution by Image Registration. Cvgip-Graph Model Im 53, pp. 231-239.

Szummer, M., Picard, R.W., 1996. Temporal texture modeling. International Conference on Image Processing, Proceedings Vol III, pp. 823-826.

Wald, L., 2002. Data Fusion: Definitions and ArchitecturesFusion of Images of Different Spatial Resolutions. Les Presses, Ecole des Mines de Paris, Paris, France.

Wang, H., Wang, J., Huang, B., 2012. Prediction for spatiotemporal models with autoregression in errors. Journal of Nonparametric Statistics 24, pp. 217-244. 